\title{
Propuesta para el uso exclusivo de la tasa interna de retorno modificada en la toma de decisión de proyectos industriales de inversión
}

\author{
Medina, J.R. ${ }^{1}$, Romero, R.L. ${ }^{2}$, y Pérez, G.A. ${ }^{3 *}$ \\ $(1,3)$ Facultad de Ingeniería Química (FIQ), \\ Universidad Nacional del Litoral (UNL), Santiago del Estero 2829, (3000) Santa Fe-Argentina; \\ Tel.: (0342)-4571164 int. 2549; Fax: (0342)- 4571162; \\ gperez@unl.edu.ar \\ (2) Instituto de Desarrollo Tecnológico para la Industria Química (INTEC-UNL-CONICET) \\ Güemes 3450; (3000) Santa Fe, Argentina
}

(Recibido/received: 08-Marzo-2013; aceptado/accepted: 28-Nov-2013)

\begin{abstract}
RESUMEN
La TIR (tasa interna de retorno) es el método de evaluación de rentabilidad de proyectos de inversión mejor conocido. Esta tasa interna de retorno representa, en la interpretación tradicional de las tasas de interés, a la tasa de interés ganada por una inversión alternativa sobre el saldo no recuperado de una inversión. Sin embargo, cuando se presenta una inconsistencia, basada en la aparición de raíces múltiples, se acude a la Tasa Interna de Retorno Modificada (TIRM) para aportar una definición a la toma de decisiones. El trabajo sostiene que este método de la TIRM puede ser utilizado siempre en la toma de decisiones económicas financieras, porque conceptualmente utiliza valores en sus variables de decisión, mucho más cercanos a los que operan en el mercado y en el conocimiento de quién toma la decisión, involucrando en su resultado el que se puede obtener con los métodos tradicionales, en el caso que estos se aplican con solvencia. Aún, este método se comprobará es efectivo en el caso de proyectos de inversión con diferentes horizontes de planificación.
\end{abstract}

Palabras clave: TIR; tasa interna de retorno; TIR modificada; proyectos de inversión.

\begin{abstract}
Internal Rate of Return (IRR) is the method of assessing profitability of investment projects better known. It represents, in the traditional interpretation, the interest rate earned on an alternative investment for the balance not recovered from that investment. However, when an inconsistency occurs, the possibility of occurrence of multiple roots, it goes to the Modified Internal Rate of Return (MIRR) to provide a definition for decision making. The work suggests that this method can always be used TIRM, in a universal way, in making financial business decisions, because conceptually used (and calculated) values of rates much closer to those operating in the market and in the knowledge of who makes the decision. In addition, this method is effective in check for investment projects with different planning horizons.
\end{abstract}

Keywords: DCF; internal rate of return; MIRR; project profitability.

\footnotetext{
${ }^{*}$ Autor para la correspondencia
} 


\section{INTRODUCCIÓN}

Es sabido que los denominados métodos de flujo de efectivo descontado son los usuales en el momento de determinar la rentabilidad de un emprendimiento o proyecto empresarial. La tasa interna de retorno puede calcularse al igualar el valor anual, presente o futuro del flujo de efectivo a 0 (cero) y resolver la raíz, que permita la igualdad (Riggs et al, 2002). O, hacer uso del más popularmente difundido VAN (Valor Actual Neto).

El presente trabajo discutirá la universalidad $\mathrm{y}$ suficiencia del método de la TIR Modificada (Tasa Interna de Retorno Modificada, o Tasa Externa de Retorno), por tener todas las variables de decisión que intervienen en el análisis y entregar un resultado confiable, y razonablemente cercano a las tasas de negocios exitosos en ejecución. La elección de la tasa, para la aplicación del método, es esencial y trascendente en la discusión aquí encarada.

\section{ANÁLISIS DE MÉTODO}

La razón que da origen a su utilización es la existencia de raíces múltiples en el polinomio resultante en la búsqueda de la tasa interna de retorno (TIR) originalmente definida. Al no cumplirse el Lema de Descartes, por haber más de un cambio de signo en los flujos de fondos anuales, el uso del método de la TIR común queda descartado, por no otorgar certeza en su resultado.

Así, para sortear esta dificultad, surge la alternativa de TIR Modificada, que presupone la definición y adopción de la llamada "tasa atractiva mínima de retorno", por parte del analista y tomador de la decisión (Eschenbach, 1995). En principio, puede decirse que esta elección está influenciada por el tipo de proyecto, la empresa que realiza la inversión, el entorno macroeconómico al momento del análisis y el criterio del analista a cargo de la misma.

En cualquier expresión de los métodos de flujo de caja descontado aparece una relación del tipo:

$$
V_{j}=V_{j}\left(F_{c j}, j=1, . N, I_{T 0}, k, N\right)
$$

La ecuación (1) representa el valor neto en el tiempo j de la relación entre los distintos flujos de fondos, para todo el horizonte de planificación $(\mathrm{N})$, con sus respectivos signos, el valor de la inversión total inicial (que se particulariza por su importancia, a pesar de representar otro flujo de fondos, este al tiempo cero inicial), el valor de la tasa de corte o costo del dinero, o costo de oportunidad y el horizonte del análisis, como se mencionó.

Estas medidas cuantitativas, tienen hipótesis no realistas que la mayoría de los textos específicos ignoran. Ambos (VAN y TIR) comparten una debilidad muy importante: ¿cómo se reinvierten los retornos?, algo inherente a la definición de los métodos de flujos de fondos descontados. Y, el valor de la TIR es el resultado de un cálculo (raíz) que tuvo en cuenta el horizonte de planificación y los flujos de fondos en los distintos tiempos (modelo discreto), por lo que no representa una tasa que signifique una oportunidad real de reinversión (Kierulff, 2008)

La ecuación representativa más general de la TIR modificada se la puede relacionar al cociente:

$\frac{(\text { Valor Futuro de ingresos netos de caja })}{(\text { Valor presente de egresos netos de caja })}$

En forma matemática se traduce en la siguiente:

$$
(1+\operatorname{TIRM})^{N} \sum_{i=0}^{N} \frac{F_{c i}^{-}}{\left(1+k_{a m}\right)^{i}}=\sum_{i=0}^{N} F_{c i}^{+}\left(1+k_{a m}\right)^{N-i}
$$

En la ecuación (3) aparecen las sumas descontadas de los flujos de fondos negativos y las sumas capitalizadas de aquellos flujos de fondos positivos, en el año que corresponda a cada caso. Pero, a su vez, de acuerdo a la ecuación (1), podemos definir un valor presente teniendo en cuenta los signos tal como se presenta, esto es: 


$$
V A N\left(k_{a m}\right)=\sum_{i=0}^{N} \frac{F_{c i}}{\left(1+k_{a m}\right)^{i}}=\sum_{i=0}^{N} \frac{\left(F_{c i}^{+}-F_{c i}^{-}\right)}{\left(1+k_{a m}\right)^{i}}=\sum_{i=0}^{N} \frac{F_{c i}^{+}}{\left(1+k_{a m}\right)^{i}}-\sum_{i=0}^{N} \frac{F_{c i}^{-}}{\left(1+k_{a m}\right)^{i}}
$$

De manera que operando sobre la ecuación (3), resulta:

$\left[\frac{(1+\text { TIRM })^{N}}{\left(1+k_{a m}\right)^{N}}-1\right] \sum_{i=0}^{N} \frac{F_{c i}^{-}}{\left(1+k_{a m}\right)^{i}}=\operatorname{VAN}\left(k_{a m}\right)$

Despejando las raíces, quedará:

$$
\frac{(1+\operatorname{TIRM})}{\left(1+k_{a m}\right)}=\left[\frac{V A N\left(k_{a m}\right)}{\sum_{i=0}^{N} \frac{F_{c i}^{-}}{\left(1+k_{a m}\right)^{i}}}+1\right]^{1 / N}
$$

En donde, para el caso particular usual, en el cual toda la inversión se realiza antes de iniciarse la operación de la planta, (método de Solomon; Park \& Sharp-Bette, 1990), es:

$$
\frac{(1+T I R M)}{\left(1+k_{a m}\right)}=\left[\frac{\operatorname{VAN}\left(k_{a m}\right)}{I_{T 0}}+1\right]^{1 / N}
$$

Ecuación que permite ver la universalidad del método. A partir de ella es posible redefinir el llamado Índice de Lucro ("Worth Index") de una manera más universal, revalorizando este intento de enlazar los criterios de VAN y de TIR, para dar coherencia a las decisiones.

Las ecuaciones (6) y (7) suponen que, para la elección del proyecto en cuestión, debe ser la TIRM mayor que ese valor de tasa atractiva mínima elegida para realizar los descuentos y capitalizaciones. Y ocurrirá siempre que:

$$
\frac{V A N\left(k_{a m}\right)}{\sum_{i=0}^{N} \frac{F_{c i}^{-}}{\left(1+k_{a m}\right)^{i}}}+1>1
$$

Y, simultáneamente debe ser:

$$
\operatorname{VAN}\left(k_{a m}\right)>0
$$

Resultado que encierra el uso del VAN, pero a una tasa base representativa del mercado y/o del negocio, elegida con todas las consideraciones económica - financieras que amerite el caso, y el riesgo percibido en su ejecución.

Por otra parte, la ecuación (8), fundamental para la conclusión anterior, no es más que el criterio empleado usando el Índice de Lucro, definido en su forma más general.

\section{DISCUSIÓN DE LA PROPUESTA}

Entonces, la pregunta esencial es: ¿qué es kam?, ese límite inferior para la aceptación de una inversión y mayormente responsable de la decisión de invertir y con características de transformar este método en universal.

Si el VAN es el origen de otras formas de evaluar rentabilidad, dando lugar a los denominados métodos de flujo de caja (o fondos) descontados, en este de la TIRM encuentra su expresión más universal, respecto a la tasa y su relación con la inversión (en realidad, con todos los flujos negativos originados por inversiones o egresos en la marcha del negocio), dando lugar a un criterio sólido ante diferentes alternativas de proyectos excluyentes $o$ proyectos únicos a definir su prosecución.

Cuando los presupuestos para inversión están restringidos a un valor tope, $\mathrm{y}$ hay proyectos excluyentes, en consecuencia, este método TIRM resulta el más indicado, si el que toma la decisión tiene precisión en la elección de la tasa mínima atractiva a usar. La tasa, ahora sí, es una real de mercado, con todas las consideraciones que demande su elección, para el caso y la empresa en particular.

Relacionado con esta variable independiente se puede ver que existen diferentes tasas de descuento para diferentes circunstancias, cosa ilustrada ampliamente 


\section{A. Balmaceda y E. Castillo}

por la bibliografía. Sus "valores" dependen de posiciones relativas, en su gran mayoría.

Si están basadas en el costo de capital su elección será por tres causas fundamentales. Esto es, impaciencia del que toma la decisión frente al futuro, costo de oportunidad y riesgo, como tercera consideración. Se puede intuir que son mayores a corto plazo, por tratarse de un futuro cercano, por ejemplo.
La universalidad del método TIRM se extiende, también, al caso de comparación entre proyectos con horizontes de planificación distintos (o vidas diferentes). Es sabido que el método más aconsejable para abordar esta toma de decisión es el Beneficio Anual (Uniforme) Equivalente (BAE), a pesar de que algunos autores sostienen que conserva el problema de repetición permanente o duración infinita (Donovan Young, 1993).

Si se tiene el caso de un proyecto con horizonte $\mathrm{M}$ y otro con $\mathrm{N}$, usando el criterio mencionado, resultará

$$
\frac{B A E(M)}{B A E(N)}=\frac{V A N_{\left(k_{a m}, M\right)}}{V A N_{\left(k_{a m}, N\right)}} \frac{\left(1+k_{a m}\right)^{M}}{\left(1+k_{a m}\right)^{N}}\left[\frac{\left(1+k_{a m}\right)^{N}-1}{\left(1+k_{a m}\right)^{M}-1}\right]
$$

Y, utilizando la expresión de las ecuación (6), se llega a la siguiente igualdad:

$$
\frac{B A E_{(M)}}{B A E_{(N)}}=\frac{\left\{\left(\frac{1+\operatorname{TIRM}}{1+k_{a m}}\right)^{M}-1\right\}}{\left\{\left(\frac{1+T I R M}{1+k_{a m}}\right)^{N}-1\right\}} \frac{\left(1+k_{a m}\right)^{M}}{\left(1+k_{a m}\right)^{N}}\left[\frac{\left(1+k_{a m}\right)^{N}-1}{\left(1+k_{a m}\right)^{M}-1}\right] \frac{\sum_{i=0}^{M} \frac{F_{c i}^{-}}{\left(1+k_{a m}\right)^{i}}}{\sum_{i=0}^{N} \frac{F_{c i}^{-}}{\left(1+k_{a m}\right)^{i}}}
$$

Que muestra, claramente, la equivalencia de ambos métodos. En consecuencia, la ecuación (11) justifica la aplicación de la TIRM para la toma de decisión entre proyectos con horizontes diferentes, con el beneficio de incorporar el peso de las distintas inversiones hechas dentro de cada horizonte de planificación.

En el peor de los casos, tiene la misma limitación que el uso del BAE, ampliamente recomendado $\mathrm{y}$ difundido, pero dando más precisión a la comparación, por lo antedicho.

Pero, esa enunciada limitación (D.Young, 1993), es solo cierta cuando $\mathrm{M}$ y $\mathrm{N}$ son mucho mayores que 1 . Es en este caso, únicamente, en donde las funciones de la tasa de descuento y el horizonte de

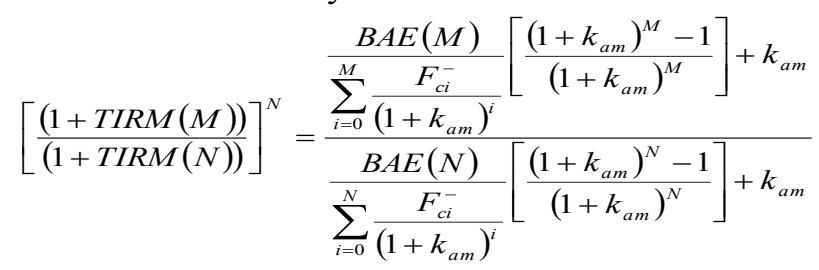

planificación, que surge de uniformizar los VAN, no corrige la diferencia de estos, por aproximarse a la unidad (tiende a uno en el límite infinito). Y, en la expresión (11), se mantiene el efecto de las inversiones, por encima de cualquier consideración de "repetición infinita", lo que valoriza la aplicación de la TIRM para estos casos. No ocurre lo mismo, tanto en el criterio del BAE, cuanto el de la TIRM, cuando $\mathrm{M}$ y $\mathrm{N}$ tiene valores no tan grandes y distintos entre sí, como es la situación que se está tratando.

Para visualizar más claramente lo antedicho se puede modificar la ecuación (1), quedando las relaciones entre TIRM de la siguiente forma: 
De modo, que este trabajo reivindica el uso de la TIRM para vidas diferentes entre proyectos, con el agregado de que es el método más adecuado para la mayoría de los casos que resultan de interés práctico.

\section{CONCLUSIONES}

La bibliografía, aún la más reciente, sigue mencionado al VAN como el método más empleado por quienes toman decisiones de futuras inversiones. Cuando este se aplica correctamente, puede usarse para evaluar proyectos por comparación entre inversiones con riesgos equivalentes.

Esto es, si aceptamos la tasa de descuento única para el procedimiento de comparación la implicancia del riesgo está implícita. Por otra parte, el costo de capital que implica esa tasa de descuento, si se modela como sugiere el método más empleado CAPM, está definida solamente por costos (distintas fuentes de dinero equivale distintos costos, etc.), lo que muestra otra limitación de esta metodología para ser universal. Por otra parte, no hay una aditividad directa entre varios VAN, como se observa en el análisis basado en la tasa definida por Fisher.

En consecuencia, si el VAN tiene estas observaciones, que conllevan limitaciones; y si la TIR tiene los problemas muy bien conocidos, que impiden darle carácter universal, este trabajo argumenta y demuestra las ventajas del uso de la TIRM y su falta de restricciones para ser utilizada universalmente en la toma de decisiones económicas financieras. Es más, esta universalidad le permite resolver inversiones con horizontes de planificación diferentes, como se ha discutido.

\section{NOMENCLATURA}

$\mathrm{Vj}=$ valor neto en el tiempo $\mathrm{j}$

$\mathrm{FC} j$ = flujo de caja del año $\mathrm{j}$ cualquiera

IT0 = inversión inicial al tiempo cero

$\mathrm{N}$ = horizonte del proyecto de inversión

$\mathrm{k}=$ tasa de descuento utilizada

$F_{c i}^{-}=$flujos de caja negativos en el año i
$F_{c i}^{+}=$flujos de caja positivos en el año i

TIRM = tasa interna de retorno modificada

$\mathrm{N}=$ horizonte de planificación del proyecto

$k_{a m}=$ tasa mínima atractiva de retorno

\section{BIBLIOGRAFÍA}

Donovan Young (1993). Modern Engineering Economy. Ed. John Wiley \& Sons, Inc.

Eschenbach, Ted G. (1995). Engineering Economy Applying Theory to Practice. Ed. IRWIN Inc.

Kierulff, H, Mirr. (2008). A better measure, Business Horizons, 51, 321-329

Park, C.S. and Sharp-Bette, G.P. (1990). Advanced Engineering Economics. Ed. John Wiley \& Sons, Inc.

Riggs, J.L., Bedworth, D.D. and Randhawa, S.U. (2002). "Ingeniería Económica", 4 Ed. Alfaomega grupo editor S.A. México.

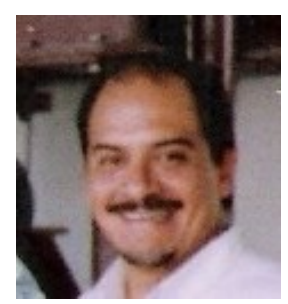

José R. Medina es Ingeniero Químico y Magister en Ciencia y Tecnología de Alimentos, recibido en la Universidad Nacional de Litoral (Argentina). Participa como Profesor de asignaturas de Ingeniería Económica y Proyecto Industrial, y con tareas de investigación sobre Tecnologías de procesamiento y conservación de matrices cárnicas.

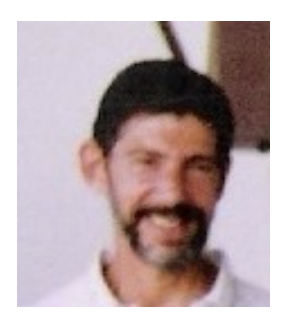

Gustavo A. Pérez es Ingeniero Químico, recibido en la Universidad Nacional del Litoral (Argentina). Desarrolla sus actividades como Profesor Responsable de las asignaturas Ingeniería Económica y Proyecto Industrial en la Facultad de Ingeniería Química. Además realiza tareas de investigación en el CONICET, en el área de "Ingeniería de Procesos" 